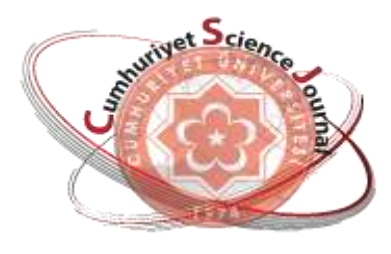

e-ISSN: $2587-246 X$

ISSN: $2587-2680$

\section{Cumanoriyet Science Journal $\cos$}

Cumhuriyet Sci. J., Vol.39-3(2018) 557-564

\title{
Streamline Topology of Axisymmetric Flow Near Non-Simple Singular Point
}

\author{
Ali DELICEOĞLU*, Deniz BOZKURT \\ Erciyes University, Science Faculty, Department of Mathematics, Kayseri, TURKEY \\ Received: 06.06.2018; Accepted: 24.07.2018 \\ http://dx.doi.org/10.17776/csj.431321
}

\begin{abstract}
The aim of this paper is to obtain streamline patterns of axisymmetric flow and their bifurcations for 2-D incompressible flows close to non-simple singular point. The streamlines of a Hamiltonian vector field system are simplified by using the homotopy invariance of the index theory. Using a homotopy invariance of the index, we develop a theory for the sufficient and necessary conditions for structural bifurcation of axisymmetric flow near non-simple degenerate critical points. The variation of parameters in the flow field can cause structural bifurcations. The bifurcation of the degenerate flow structure is obtained when it is perturbed slightly.
\end{abstract}

2010 Mathematics Subject Classification: 34D, 35Q35, 58F76, 86A10.

Keywords: Structural stability, divergence-free vector field, Lagrangian dynamics, structural bifurcation, singularity classification.

\section{Basit Olmayan Dejenere Nokta Civarındaki Eksenel Simetrik Akış Topolojisi}

\begin{abstract}
Özet. Bu makalenin amacı basit olmayan tekil nokta civarındaki 2-Boyutlu sıkıştırılamaz akışlar için eksenel simetrik akışların akış çizgi modellerini ve onların çatallanmalarını elde etmektir. Hamiltoniyen vektör alan sisteminin akış çizgileri, indeks teorisinin homotopi değişmezliği kullanılarak basitleştirildi. İndeksin homotopi değişmezliği kullanılarak, basit olmayan dejenere nokta civarında, eksenel simetrik akışın yapısal çatallanması için yeterli ve gerekli koşullar için bir teori geliştirildi. Akış alanındaki parametrelerin değişimi yapısal çatallanmalara neden olabilir. Dejenere akış yapısının çatallanması, bu parametrelerin hafifçe değiştirilmesiyle elde edildi.
\end{abstract}

Anahtar Kelimeler: Yapısal kararlılık, divergense-serbest vektör alanı, Lagrange dinamiği, yapısal çatallanma, tekillik sınıflandırması.

\section{INTRODUCTION}

In the present paper, we are interested in the structural bifurcation of axisymmetric flow close to nonsimple singular points. Brøns et. al. in [1] studied topology of vortex breakdown bubbles in a cylinder with a rotating bottom and a free surface. They obtained possible flow bifurcations of streamline patterns in axisymmetric flow. Brøns et. al. in [2] have also considered the steady vortex breakdown in axisymmetric flow. Bisgaard et. al. in [3] have observed the off-axis ring in a cylinder. Further results on flow topology in axisymmetric flow can be found in the papers [4-6].

Different methods can be used to obtain qualitative features of fluids flows. A singular point analysis is performed by Bakker [7]. He obtains detailed information about the local flow topology. Brøns et. al. in 
[8] and Hartnack [9] used normal form transformation to study streamline topology near simple degenerate critical points near and away from boundaries. Normal form transformation determines the codimension of degeneracies. This technique was also used by Deliceoğlu and Gürcan [10-12]. They investigated flow topology near a non-simple degenerate singular point.

Structural bifurcation of two-dimensional incompressible flow was studied by Ma and Wang [13]. They obtained bifurcation near degenerate points with zero index. They studied topological structure of divergence-free vector fields, and its evaluation in time. Topological ideas have been applied in several other studies in the investigation of structural stability and topology of divergence free-vector fields (see e.g. $[14,15]$.

The scope of the present paper is to make connections between the index of axisymmetric vector field $u(., t)$ near a non-simple degenerate critical point and its Taylor expansion. The number of free parameters in the Taylor expansion of a divergence-free vector field is reduced by using the homotopy invariance of the index. This method significantly reduces the computational cost of finding a normal form of stream function preserving the flow structure under the transformation. This method was also used by the authors $[13,14]$. In this study, the works $[3,12]$ will be revisited through the index theory, as will be seen, structural classification of divergence-free vector fields near the non-simple critical points is simplified significantly.

\section{FORMULATION OF THE PROBLEM}

Axisymmetric flows can be described using cylindrical coordinates $(r, \theta, z)$ with the corresponding velocity field $(u, v, w)$ depending only on $r, z$. In the works [1-3], axisymmetric flows can be constructed as follows:

$$
u=\frac{1}{r} \frac{\partial \psi}{\partial z}, v=r \frac{d \theta}{d t}, w=-\frac{1}{r} \frac{\partial \psi}{\partial r}
$$

The differential equations for the contours can be transformed into Hamiltonian system by using $\rho=r^{2} / 2$. Then the equation (1) becomes

$$
\dot{\rho}=r u=\frac{\partial \psi}{\partial z}, \dot{z}=w=-\frac{\partial \psi}{\partial \rho} .
$$

$\psi$ is expanded in a power series,

$$
\psi=\sum_{n+m=0}^{\infty} a_{i, j} \rho^{n} z^{m}
$$

We have the boundary conditions

$$
\psi(\rho, z)=\psi(\rho,-z), \quad \psi=0, \frac{\partial^{2 n-1} \psi}{\partial r^{2 n-1}}=0 \text { for } r=0 \text { and all } n \in N .
$$

By applying boundary condition (4) to the Taylor coefficients in (3), we get the stream function

$$
\psi=a_{1,0} \rho+a_{2,0} \rho^{2}+a_{3,0} \rho^{3}+a_{1,2} \rho z^{2}+a_{4,0} \rho^{4}+a_{2,2} \rho^{2} z^{2}+O\left((\rho, z)^{5}\right),
$$

and substituting this in the equation (2), we obtain the level curves of $\psi$ as follows: 


$$
\begin{aligned}
& \dot{\rho}=\rho\left(a_{1,2} z+2 a_{2,2} \rho z+\ldots\right) \\
& \dot{z}=-\left(a_{1,0}+2 a_{2,0} \rho+3 a_{3,0} \rho^{2}+a_{1,2} z^{2}+\ldots\right) .
\end{aligned}
$$

If $a_{1,0}=0$ in (6) the origin is a critical point. If $a_{2,0}=0$ in (6), the critical point becomes a simple degenerate critical point which was investigated by Brons et. al. [1]. Here using the index theory, flow topology near the non-simple singular point is characterized with the assumptions $a_{2,0}=0$. From the assumption and the boundary conditions, we can write the stream function as follows:

$$
\psi=a_{3,0} \rho^{3}+a_{1,2} \rho z^{2}+a_{4,0} \rho^{4}+a_{2,2} \rho^{2} z^{2}+a_{5,0} \rho^{5}+a_{3,2} \rho^{3} z^{2}+a_{1,4} \rho z^{4}+O\left((\rho, z)^{6}\right) .
$$

The stream function (7) leads to a dynamical system

$$
\begin{aligned}
& \dot{\rho}=2 a_{1,2} \rho z+2 a_{2,2} \rho^{2} z+2 a_{3,2} \rho^{3} z+4 a_{1,4} \rho z^{3}+O\left((\rho, z)^{5}\right), \\
& \dot{z}=-a_{1,2} z^{2}-a_{3,0} \rho^{2}-4 a_{4,0} \rho^{3}-2 a_{2,2} \rho z^{2}-5 a_{5,0} \rho^{4}-3 a_{3,2} \rho^{2} z^{2}-a_{1,4} z^{4}+O\left((\rho, z)^{5}\right) .
\end{aligned}
$$

The dynamical system (8) corresponds to a $2 D$ divergence-free vector fields $u^{0}(\rho, z)=u\left(\rho, z, t_{0}\right)=\left(u_{1}\left(\rho, z, t_{0}\right), u_{2}\left(\rho, z, t_{0}\right)\right)$ on $M$ is a closed and bounded domain. Then we write the vector field $u^{0}(\rho, z)$ as follows:

$$
u^{0}(\rho, z)=\left\{\begin{array}{l}
2 \alpha \rho z+o\left((\rho, z)^{2}\right), \\
-\alpha z^{2}-f(p)+o\left(z^{2}\right)
\end{array}\right.
$$

where $\alpha \neq 0$ and $f(\rho)=\sum_{k=0}^{\infty}(k+1) \beta \rho^{k}(k \geq 2$ and $\beta \neq 0)$.

\section{DEGENERATE SINGULARITIES OF AXISYMMETRIC VECTOR FIELD}

We consider the relations between the index of $u^{0}$ at $\bar{x}$ and coefficients of $u^{0}$ in its Taylor expansion close to non-simple degenerate point $\bar{x} \in M$. Let $\bar{x} \in M$ be a non-simple degenerate singular point of $u^{0}(\rho, z)$, and

$$
\begin{aligned}
& D u^{0}(\bar{x})=0, \\
& \frac{\partial^{k}\left(u_{2}\right)}{\partial^{k} \rho} \begin{cases}=0, & k<2, \\
\neq 0 & k \geq 2 .\end{cases}
\end{aligned}
$$

Under the above conditions, the vector field is written by

$$
u^{0}(\rho, z)=\left\{\begin{array}{l}
2 \alpha \rho z+o\left((\rho, z)^{2}\right) \\
-\alpha z^{2}-(k+1) \beta \rho^{k}+o\left(z^{2} \rho^{k}\right) .
\end{array}\right.
$$


Lemma 1: Let $\bar{x} \in M$ be an isolated degenerate singular point of $u^{0}$ satisfying (12). In a small neighborhood of $\bar{x}$, the index of $u$ is stated as follows:

ind $\left(u^{0}, x_{0}\right)=\left\{\begin{aligned} 0 & \text { as } k=\text { even and } \alpha \beta>0 \text { in }(12), \\ -1 & \text { as } k=\text { even and } \alpha \beta<0 \text { in (12), } \\ -1 & \text { as } k=\text { odd in (12). }\end{aligned}\right.$

Proof of Lemma 1: Let

$u_{t}^{0}(\rho, z)=\left\{\begin{array}{l}2 \alpha \rho z+t o\left((\rho, z)^{2}\right), \\ -\alpha z^{2}-(k+1) \beta \rho^{k}+t o\left(z^{2} \rho^{k}\right),\end{array}\right.$

where $0 \leq t \leq 1$. There exist a neighborhood $U \subset M$ of $\bar{x}(=0)$, such that $u_{t}(\rho, z)$ has a stagnation point $\bar{x}=0$ in $U$ for all $0 \leq t \leq 1$. Using the homotopy invariance of the index, one obtains

$$
\operatorname{ind}\left(u_{0}, \bar{x}\right)=\operatorname{ind}\left(u_{1}, \bar{x}\right)=\operatorname{ind}(u, \bar{x})
$$

In a small neighborhood of $\bar{x}=0$ the flow patterns of $u_{0}(\rho, z)=\left(2 \alpha \rho z,-\alpha z^{2}-(k+1) \beta \rho^{k}\right)$ are given by the following equations:

$$
\rho\left(\alpha z^{2}+\beta \rho^{k}\right)=C, 0 \leq|C| \leq \delta
$$

Here $\alpha, \beta \neq 0$ and $k \geq 2$. We can see from (14) that if $k$ is even and $\alpha \beta>0$ then the orbits of $u_{0}$ is as illustrated in Fig. 1 (a). If $k=2$ and $\alpha \beta<0$ then the orbits of $u_{0}$ is as illustrated in Fig. 1 (b). If $k$ is even, $k>2$ and $\alpha \beta<0$ then the orbits of $u_{0}$ is as illustrated in Fig. 1 (c). In a small neighborhood of $\bar{x}=(0,0)$, if $k$ is odd and $\alpha \beta<0$ then the orbits of $u_{0}$ is as illustrated in Fig. 1 (d).

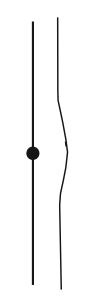

(a)

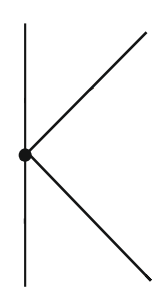

(b)

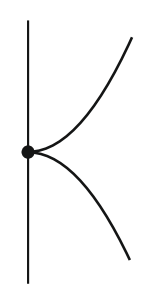

(c)

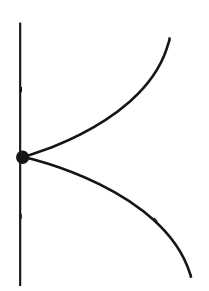

(d)

Figure 1. Non-simple degenerate singular points of $u$ given by (12). (a) A degenerate point with ind $\left(u^{0}, x_{0}\right)=0$ for the case where $k$ is even and $\alpha \beta>0$. (b) A degenerate saddle point with ind $\left(u^{0}, x_{0}\right)=-1$ for case where $k=2$ and $\alpha \beta<0$. (c) A degenerate saddle point with ind $\left(u^{0}, x_{0}\right)=-1$ for the case where $k$ is even, $k \geq 4$ and $\alpha \beta<0$. (d) A degenerate cusp point with ind $\left(u^{0}, x_{0}\right)=-1$ for the case where $k$ is odd and $\alpha \beta<0$. 


\section{UNFOLDING OF A NON-SIMPLE DEGENERATE CRITICAL POINT FOR THE AXISYMMETRIC FLOW}

In this section we consider flow bifurcation of a divergence free vector field close to a non-simple degenerate interior point in axisymmetric flow. To study the bifurcations close to the non-simple degenerate critical points, we consider the Taylor expansion of axisymmetric vector field $u(\rho, z, t)$ at $t_{0}\left(0<t_{0}<T\right)$ :

$$
\begin{aligned}
& u(x, t)=u^{0}(x)+\left(t-t_{0}\right) u^{1}(x)+o\left(\left|t-t_{0}\right|^{2}\right), \\
& u^{0}(x)=u\left(x, t_{0}\right), \\
& u^{1}(x)=\frac{\partial}{\partial t} u\left(x, t_{0}\right) .
\end{aligned}
$$

We start with the following condition.

Assumption (1): Let $\bar{x} \in M$ an isolated degenerate singular point of $u^{0}(x)$ satisfying the condition of Lemma 1. Suppose that

$$
\begin{aligned}
& D u(\bar{x})=0, \\
& u^{1}(\bar{x}) \cdot e_{2} \neq 0,
\end{aligned}
$$

where $e_{2}$ is the unit vector and we assume that $u^{0} \in C^{n}$ close to $\bar{x} \in M$ for $k \geq 2$,

$$
\frac{\partial^{k}\left(u_{2}\right)}{\partial^{k} \rho}\left\{\begin{array}{l}
=0, \text { for } k<2, \\
\neq 0 \text { for } k \geq 2
\end{array}\right.
$$

Theorem 1: Let the axisymmetric vector field $u$ satisfy all conditions of Assumption (1) with index $=0$.

Then, $u(x, t)$ evaluates from $\left(\bar{x}, t_{0}\right)$ three non-degenerate stagnation points in a small neighborhood of $\bar{x} \in M$ for any $t>t_{0}$, two of which are saddle points and another one is a center. In a small neighborhood of $\bar{x}, u(x, t)$ has no singular point for $t<t_{0}$.

Proof of Theorem 1 From Assumption (1) and Lemma 1 the vector field $u^{0}(\rho, z)$ has the Taylor expansion at $\bar{x}=0$ as follows:

$$
u^{0}(\rho, z)=\left\{\begin{array}{l}
2 \alpha \rho z+o\left((\rho, z)^{2}\right), \\
-\alpha z^{2}-(k+1) \beta \rho^{k}+o\left(z^{2} \rho^{k}\right) .
\end{array}\right.
$$

where $\alpha \neq 0, \beta \neq 0$. From Lemma 1, if the index $=0$ then $k \geq 2, k$ is even and $\alpha \beta>0$. By the condition (17) we can take 


$$
u^{1}(\rho, z)=\left\{\begin{array}{l}
O(\rho, z) \\
b_{0}+O(\rho, z)
\end{array}\right.
$$

where $b_{0} \neq 0$. We assume that $\alpha>0, \beta>0$ (or $\alpha<0$ and $\beta<0$ ) and $b_{0}>0$. In this case it is easy to see that $u^{0}-\varepsilon u^{1}$ has no singular point in the physical plane. Now we consider the singular points of the vector field $u^{0}+\varepsilon u^{1}$ for all $\varepsilon>0$ sufficiently small.

$$
\begin{aligned}
& 2 \alpha \rho z+o(\rho, z)^{2}+\varepsilon O(\rho, z)=0, \\
& -\alpha z^{2}-(k+1) \beta \rho^{k}+o\left(z^{2} \rho^{k}\right)+\varepsilon b_{0}+\varepsilon O(\rho, z)=0 .
\end{aligned}
$$

The equation (21) is satisfy for $\rho=0$ and the solutions of (22) in term of $z$ is as follows:

$$
z= \pm\left(\frac{\varepsilon b_{0}}{\alpha}\right)^{1 / 2}
$$

Similarly, equation (21) is satisfy for $z=0$ and the solutions of (22) in term of $z$ is as follows:

$$
\rho= \pm\left(\frac{\varepsilon b_{0}}{(k+1) \rho}\right)^{1 / k} \text {. }
$$

From this, we get the solutions of $u^{0}+\varepsilon u^{1}$ as follows:

$$
P_{1,2}=\left(0, \pm\left(\frac{\varepsilon b_{0}}{\alpha}\right)^{1 / 2}\right)+o\left(\varepsilon^{1 / 2}\right), \quad P_{3,4}=\left( \pm\left(\frac{\varepsilon b_{0}}{(k+1) \rho}\right)^{1 / k}, 0\right)
$$

Finally, we shall show that these critical points are non-degenerate. The determinant of the Jacobian matrix gives us that

$$
\begin{aligned}
\operatorname{det} D\left(u^{0}+\varepsilon u^{1}\right) & =\left(\frac{\partial}{\partial x}\left(u_{1}^{0}+\varepsilon u_{1}^{1}\right)\right)^{2}-\frac{\partial}{\partial y}\left(u_{1}^{0}+u_{1}^{1}\right) \cdot \frac{\partial}{\partial x}\left(u_{2}^{0}+\varepsilon u_{2}^{1}\right) \\
& =-4 \alpha^{2} z^{2}+2 \alpha \beta(k+1) \rho^{k},
\end{aligned}
$$

which yields that

$$
\left.\operatorname{det} D\left(u^{0}+\varepsilon u^{1}\right)\right|_{P_{1,2}}<0,\left.\quad \operatorname{det} D\left(u^{0}+\varepsilon u^{1}\right)\right|_{P_{3,4}}>0 .
$$

Thus, it is proven that $u^{0}+\varepsilon u^{1}$ has at least four singular point for any sufficiently small $\varepsilon>0 . P_{1,2}$ are saddle points and $P_{3,4}$ are center points. But the only three of them appear on the right side of the axisymmetric flow. The complete bifurcation diagram is as shown in Fig. 2 (a). That finishes the proof of Fig. 1(a). The other cases are proved similarly. We omit the detail of computation. The unfolding of the non-simple degenerate points is given in Fig 2(b) and Fig. 2(c). 
a)

b)

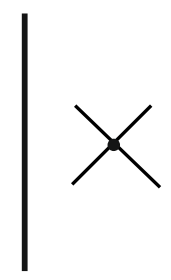

$\mathrm{t}<\mathrm{t}_{0}$

c)

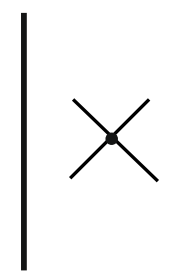

$\mathrm{t}<\mathrm{t}_{\mathrm{n}}$

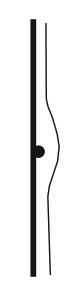

$t=t_{0}$

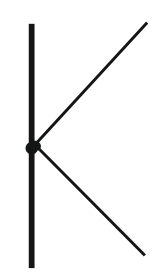

$\mathrm{t}=\mathrm{t}_{0}$

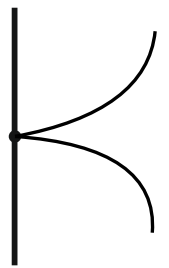

$\mathrm{t}=\mathrm{t}_{0}$

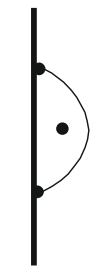

$t>t_{0}$

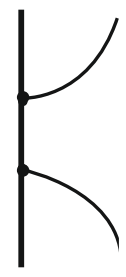

$t>t_{0}$

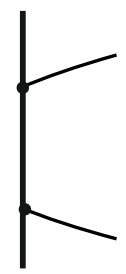

$t>t_{0}$

Figure 2. Schematic illustration of structural bifurcation diagram for Lemma 1.

\section{CONCLUSION}

In this paper, streamline topology of axisymmetric flow near the non-simple-degenerate singular points are investigated from a topological point of view. Using a homotopy invariance of the index we develop a theory for the sufficient and necessary conditions for structural bifurcation of axisymmetric flow near non-simple degenerate singular points. The approach is based on the application of the homotopy invariance of the index theory to the stream function. As we build theoretical tools in two-dimensional axisymmetric flow, we obtain new flow pattern.

\section{REFERENCES}

[1]. Brøns, M., Voigt, L.K. and Sørensen, J.N., Topology of vortex breakdown bubbles in a cylinder with a rotating bottom and a free surface, J. Fluid Mech. 428 (2001) 133-148.

[2]. Brøns, M., Voigt, L.K. and Sørensen, J.N., Streamline topology of steady axisymmetric vortex breakdown in a cylinder with co- and counter-rotating end-covers, J. Fluid Mech., 401 (1999) 275292.

[3]. Bisgaard, A.V., Brøns, M. and Sørensen, J.N., Vortex breakdown generated by off-axis bifurcation in a cylinder with rotating covers, Acta Mech. 187 (2006) 75-83.

[4]. Brøns M., Topologic al fluid mechanics of axisymmetric flows, In simulation and identification of organized structures in flows, (ed. J. N. Sørensen et al.), Kluwer Academic Publishers, Dordrecht, (1999) 213-222. 
[5]. Brøns M., Streamline patterns and their bifurcations using methods from dynamical systems, In: Ricca R.L. (eds), An introduction to the geometry and topology of fluid flows, NATO Science Series (Series II: Mathematics, Physics and Chemistry), Springer, Dordrecht, 47 (2001) 167-182.

[6]. Bisgaard, A. V., Structures and bifurcations in fluid flows with applications to vortex breakdown and wakes, PhD thesis, Department of Mathematics, Technical University of Denmark, (2005).

[7]. Bakker, P.G., Bifurcation in flow patterns, vol. 2: Nonlinear topics in the mathematical sciences, Dordrecht: Klüver, (1991).

[8]. Brøns, M. and Hartnack, J.N., Streamline topologies near simple-degenerate critical points in twodimensional flow away from boundaries, Phys. Fluids, 11(1999) 314-324.

[9]. Hartnack, J.N., Streamlines topologies near a fixed wall using normal form, Acta Mech., 136 (1999) 55-75.

[10]. Deliceouğlu, A. and Gürcan, F., Streamline topology near non-simple degenerate critical points in two-dimensional flow with symmetry about an axis, J. Fluid Mech., 606 (2008) 417-432.

[11]. Gürcan F. and Deliceoğlu, A., Streamline topologies near non-simple degenerate points in two dimensional flows with double symmetry away from boundaries and an application, Phys. Fluids, 17 (2005) 093116.

[12]. Deliceoğlu, A., Dinamik sistemler ve basit olmayan dejenere nokta civarındaki sıkıştırılamaz akışların topolojik çatallanmaları, Ph.D Thesis, University of Erciyes, (2004).

[13]. Ma, T. and Wang, S., Interior structural bifurcation and seperation of 2D incompressible flows, J. Math. Phys., 45 (2004) 1762-1776.

[14]. Ma, T. and Wang, S., Geometric theory of incompressible flows with applications to fluid dynamics (Mathematical Surveys and Monographs, American Mathematical Society), (2005).

[15]. Ma, T. and Wang, S., Structural classification and stability of divergence-free vector fields, Physica D, 171 (2002) 107-126. 